\title{
PRACTICE
}

\section{A Model Interdisciplinary Collaboration to Engage and Mentor Underrepresented Minority Students in Lived Arctic and Climate Science Research Experiences}

\author{
Arnell Garrett, Delmarva Analysis \\ Frances D. Carter-Johnson, National Science Foundation \\ Susan M. Natali, Woodwell Climate Research Center \\ John D. Schade, Woodwell Climate Research Center \\ Robert Max Holmes, Woodwell Climate Research Center
}

\begin{abstract}
The Polaris Project, a National Science Foundation-funded program at the Woodwell Climate Research Center, aims to comprehensively address minority participation in climate and Arctic science research. The project implemented design principles to recruit, motivate, and retain African Americans, Hispanics, Native Americans or Alaskan Natives, and women through immersive, field research experiences. The project included undergraduate and graduate students from environmental science, ecology, hydrology, biology, forestry, and geology. Ninety-five percent of participants identified as African American, Hispanic, Native American or Alaskan Native, and/or female. Critical participant outcomes included development of interdisciplinary research projects, involvement in self-efficacy and advocacy experiences, and increased awareness and discussion of Arctic research careers. All outcomes contributed to the Polaris Project's role as a model climate science research program.
\end{abstract}

Keywords: Arctic science, immersive field research, interdisciplinarity, minority students, self-efficacy, underrepresented students

doi: $10.18833 /$ spur/5/1/4

International, national, state, and local communities are facing increases in hazardous natural events due to climate changes. Responding to these events requires increased knowledge, innovation, collaboration, advocacy, activism, and an overall increase in participation in climate change research to effectively combat this global problem. This study focuses on the Polaris Project, a National Science Foundation-funded program that examines climate change in the Arctic. The Arctic is considered the epicenter of global climate change as evidenced by greater warming and the region's particular sensitivity to and influence on climate warming (Pastick at al. 2015; Saito et al. 2013). Despite the importance of understanding the perils of Arctic warming and climate change for human existence, research shows that the United States currently has a population of undergraduate and noncollege-educated citizens that is largely illiterate in the geosciences. This situation limits public understanding of Arctic research findings and global climate implications (Huntoon and Lane 2007; Peppoloni and Di Capua 2016). The lack of understanding is further evidenced by the fact that the field of geosciences has consistently awarded the fewest degrees for STEM disciplines across all academic levels (Callahan et al. 2017; Huntoon and Lane 2007; McDaris et al. 2017; Sherman-Morris and McNeal 2016; Wolfe and Riggs 2017). Huntoon and Lane posit that interdisciplinary and cultural diversification of students matriculating into Arctic and climate change graduate studies could improve the ability of the geosciences to communicate information across diverse populations.

To implement a comprehensive solution to the problem of lack of interest and illiteracy in the geosciences among diverse undergraduate STEM majors, researchers at the Woodwell Climate Research Center redesigned 
FIGURE 1. Polaris Project Multiyear Plan for Cohort-Based Extended Research Experience

Annual Expedition Cycle - Current Year Participants

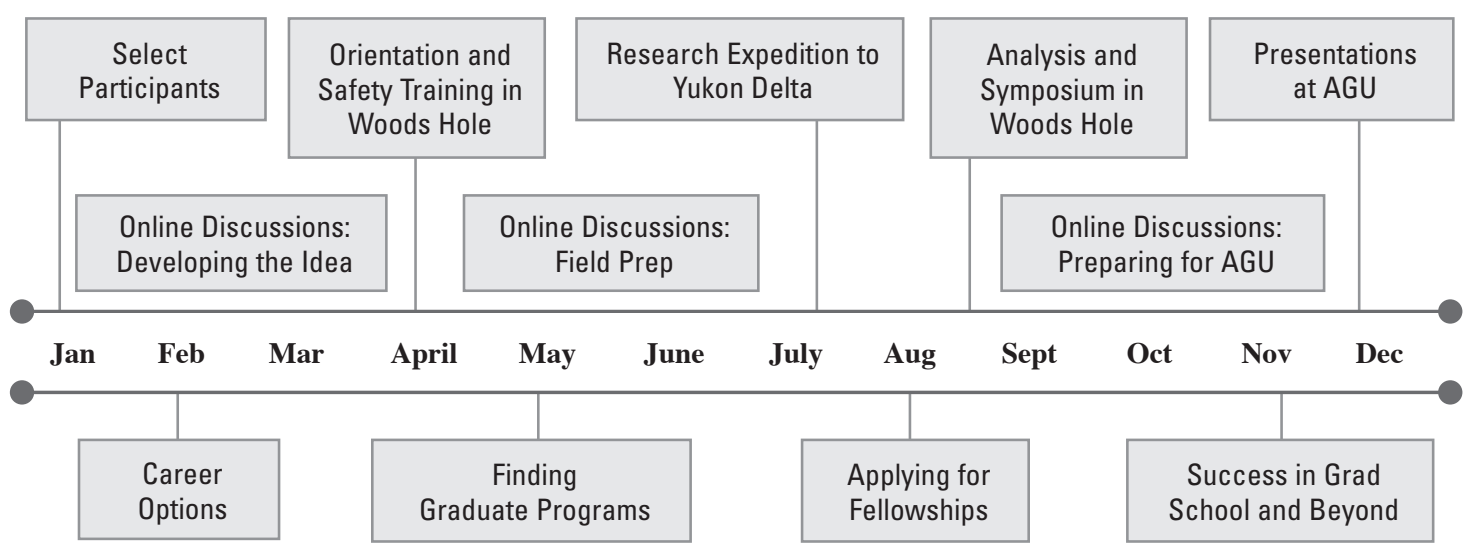

Ongoing Professional Development Webinars - All Participants

the Polaris Project in 2016. The budget, logistics, and recruitment-driven redesign focused on moving the field research experience from Siberia to Alaska to allow recruitment of undergraduate and graduate students from underrepresented groups who might be more open to a domestic research location (Garrett and Carter-Johnson 2019). The Polaris Project was designed to catalyze a change in the racial and ethnic demographics of Arctic science by engaging diverse students and faculty in cutting-edge Arctic research and providing extended research and mentoring opportunities to support the pursuit of Arctic studies. The project also aimed to increase racial and ethnic diversity among Arctic researchers within the US geoscientific workforce through interdisciplinary research collaborations between student researchers and faculty focused on climate change research. This article presents the Polaris Project as an innovative model of research participation designed to engage diverse communities. Polaris participants were encouraged to develop interdisciplinary climate change solutions based on their lived experiences with hopes of increasing their understanding of and participation in combating climate change.

\section{Polaris Project Overview}

To address concerns of underrepresentation in climate change research broadly and Arctic science more specifically, Polaris principal investigators (PIs) developed the Polaris Project, a cohort-based extended research experience. As outlined in Figure 1, an annual research expedition to the Yukon Kuskokwim Delta (YKD), Alaska, was the centerpiece of the experience. Additionally, the Polaris Project included in-person and virtual professional development opportunities such as virtual research team meetings and group attendance at the American Geophysical
Union (AGU), the annual international conference for geoscience. These activities were aimed at (1) building research experiences and community for each cohort of Polaris participants and (2) providing students from underrepresented groups with the support needed to maintain interest and inclusion in climate change research fields. The following section provides details about the intent and implementation of the Polaris Project design principles.

\section{Design Principle 1 (DP1): Recruiting and Retaining Diverse Participants and Building Capacity for Effective and Extended Mentoring}

Recruiting diverse participants was built into the communication and application aspects of the Polaris Project. As shown in Table 1, the program announced the opportunity at historically Black colleges and universities; tribal colleges and universities and Native American-serving institutions; Hispanic-serving institutions; to science diversity groups; and to program officers of federal programs mandated with increasing diversity in STEM and the geosciences. Applicants to the program completed personal statements and obtained letters of recommendation, which described the following: (1) applicants' abilities to work well in a diverse group, (2) applicants' abilities to conduct remote field research, (3) applicants' abilities to maintain commitment when faced with challenges, (4) applicants' abilities to develop team working skills, and (5) applicants' interest in graduate-level studies in Arctic science-related disciplines. Using similar outreach techniques, Polaris PIs formed relationships with faculty at minority-serving institutions and recruited visiting faculty to serve as mentors in the Polaris Project.

Upon solidifying the cohort of students and visiting faculty mentors, the Polaris PIs hosted orientation at the 
TABLE 1. Polaris Project Recruitment Emails by Institution Type and Program Year

\begin{tabular}{|l|c|c|c|}
\hline Institution type & \multicolumn{2}{|c|}{ Program year } & \\
\hline & 2017 & 2018 & $\%$ of Average \\
\hline Historically Black college or university & 14 & 17 & $34 \%$ \\
\hline Native American-serving institutions & 13 & 9 & $24 \%$ \\
\hline Other type of college or university & 8 & 7 & $16 \%$ \\
\hline $\begin{array}{l}\text { Science Diversity Groups - unaffiliated with } \\
\text { a college or university }\end{array}$ & 4 & 5 & $10 \%$ \\
\hline NSF Diversity Program directors & 7 & 1 & $9 \%$ \\
\hline Hispanic-serving institutions & 3 & 3 & $7 \%$ \\
\hline Total & 49 & 42 & $100 \%$ \\
\hline
\end{tabular}

Woodwell Climate Research Center each April. Participants met mentors and other members of the cohort, learned about Arctic safety for the field research expectation, and discussed requirements for American Geophysical Union presentations. Participants also toured the Woodwell Climate Research Center, visited the center's research laboratories, received training for analytic instruments, practiced installing tents, and practiced safety exercises. Mentors and students engaged in research discussions at orientation and virtually via monthly online meetings about assigned and self-identified journal articles and participated in field expeditions to increase exposure to current permafrost research. The goals for these activities were to educate participants about how all STEM disciplines relate to permafrost and climate change and to stimulate their thinking about independent research questions.

The mentoring component of DP1 focused on building capacity for effective and extended mentoring. This component derived its foundation in the research mentoring literature as well as from the expertise of the PIs who designed and conducted research experiences. Research suggests that undergraduate student-faculty mentoring relationships often involve and are facilitated by graduate students and postdoctoral students (Atkins 2020; Joshi, Aikens, and Dolan 2019; Nicholson et al. 2017). Mentoring by several mentors that extends beyond a summer or academic year research experience also is recommended (Bradley et al. 2017; National Academies of Sciences, Engineering, and Medicine 2020; Nicholson et al. 2017). Polaris participants were offered a multiyear and multi-mentor research experience opportunity. However, this article only reports on mentoring that occurred in year 1 .

Arctic scientists from varied backgrounds served as mentors for student participants. Mentors' research foci included studies of vegetation, aquatic ecosystems, greenhouse gases and permafrost thaw, and wildfires. The wide range of mentor expertise was critical for two reasons. First, given the complexity involved in investigating ancient carbon storage in the permafrost, multidisciplinary perspectives were needed. Second, participants received mentoring from scientists with interdisciplinary research experience, which enhanced understanding of the implications of permafrost thaw in this region for local and global climate systems. More details about the activities of the interdisciplinary research program are discussed in the next section, "Design Principle 2."

Mentoring in the Polaris Project was primarily conducted as group mentoring, allowing groups of mentors to respond to the research-related questions posed by participants. Polaris PIs served as faculty mentors for each Polaris cohort. At least one mentor from an underrepresented group also was included on the Polaris Project team each year. Although the Polaris faculty mentors remained consistent across cohorts, visiting faculty changed with each cohort but remained connected to the year's cohort of students for the entire year, from orientation to presentation at AGU.

Previous mentoring experiences of Polaris PIs indicated the need to build collaborative teams with both undergraduate and graduate students around specific research projects. Because the interdisciplinary nature of climate science allows pursuit of Arctic science graduate education as well as research and career opportunities for those with various undergraduate science degrees, graduate students both benefited as early career scientists and contributed to mentoring undergraduates through the collaborative research teams. Polaris Project graduate student participants, like the undergraduate participants, lacked previous participation in an interdisciplinary field experience in Alaska. Their research experiences with Polaris provided an immersive field experience, practice with writing analyses, and extended mentoring opportunities. 


\section{Design Principle 2 (DP2): Providing an Immersive and Interdisciplinary Field-Based Research Experience}

Although recruiting and retaining diverse scholars and mentors was important to the Polaris Project, advancing scientific knowledge in climate science also was paramount. As such, the immersive and interdisciplinary field research-based experience, the centerpiece of the Polaris Project, often overlapped with or was the backdrop for the intentional recruitment and extended mentoring efforts. Polaris PIs identified, from previous Polaris expeditions, that the greatest impact on the team and the best research outcomes occurred when students and mentors explored new areas together. This novel exploration allowed both students and faculty to share in and benefit from the excitement of exploring a new and relatively understudied environment. As part of their exploration experiences, students learned how to read a landscape and identify geographic patterns by modeling the research activities of their faculty mentors. Additionally, the PIs found that students benefited most intellectually and produced the best research outcomes when they were allowed to take ownership of a research idea. A central tenet of the expedition, facilitated by the online scientific sessions and the orientation research article review, was for students to develop an independent research-based project. Polaris participants were guided to develop research projects with a focus on their disciplines as well as their scientific and cultural interests. Although Polaris PIs discussed technical needs and climate change implications of the studentdeveloped projects with participants, the objective was to allow previous scientific and cultural experiences guide participants' research projects. The goal was for deep intellectual immersion into a scientifically and socially urgent topic that would resonate with students, build their awareness and exposure to interdisciplinary thinking, and instill a sense of accomplishment with climate change research. As another objective, the Polaris Project aimed to motivate and sustain participation in climate or Arctic science research careers. In summary, the Polaris Project considered the student-developed research projects as central to advancing scientific knowledge in climate science and preparing students for careers in climate or Arctic science. The following section outlines other aspects of the research and field experience design that make the comprehensive Polaris Project a vital model for climate change research and advocacy.

Literature on diversity in climate change research suggests cultural immersion in and consideration of marginalized cultures as opposed to assimilation into traditional research. Opportunities to facilitate forums for underrepresented minority researchers and students to share their perspectives on science and culture during traditional research activities are critical to increasing inclusivity in geoscience education (Adetunji et al. 2012; Callahan et al. 2015, 2017; Mattheis, Murphy, and Marin-Spiotta 2019). The Polaris Project intentionally allotted time for both mentors and mentees to share scientific and cultural thoughts and experiences during orientation and travel together to the expedition site and allowed the cohort to bond and build relationships outside of science. Prior to the field experience, mentors and mentees participated in climate change meetings with Alaskan Native community members, provided briefs on anticipated research questions, and received feedback on their research questions and ideas.

The immersive field experience was designed to provide an intellectually, culturally, and physically stimulating experience for student participants. Participants flew via float plane to the YKD camp, where they resided and collected data for 14 days. The isolated camp was located on the tundra, a semipermanent landmass consisting of rivers, ponds, and a complicated network of wetlands, abundant vegetation, and wildlife.

A major impact of climate change in Alaska, the YKD, and other regions of the Arctic is the melting of permafrost, the frozen layer beneath the tundra. Melting of the permafrost is critical to climate change because of uncertainties associated with the amount of carbon released into the atmosphere. These uncertainties result from limited information on the size and vulnerability of Arctic carbon pools. To investigate these uncertainties, Polaris Project participants from different disciplines designed studentdeveloped research projects as part of the immersive field experience. Polaris PIs and participants worked on collaborative research teams to make fundamental scientific discoveries related to the vulnerability of permafrost carbon in the Yukon River Delta.

During the expedition, membership in collaborative research groups changed daily. Program participants were paired with different mentors and two to three different students daily to collect the data necessary to answer their student-developed research questions. This pairing exposed students to differing strategies for data collection in climate change research. Collaborative teams were expected to support data collection of each team member by collecting water samples, soil samples, and leaf or plant samples for three respective participants. On the following day, these three participants would be assembled into a different group with a different mentor. Participants were not exclusively paired with an individual mentor and were encouraged to find time to work with all available mentors to learn diverse research techniques that could be used to answer their research questions. However, based on student interests, each faculty member oversaw the research of specific students. This collaborative data collection approach facilitated interdisciplinary research for participants and mentors. All participants were allowed 
opportunities to discuss and understand research projects from different disciplines by supporting fellow participants in their projects.

After returning from the YKD, students analyzed their data at the Woodwell Climate Research Center for 14 days. During these two weeks, group mentoring continued to be driven by research questions and analytic techniques. Polaris PIs and visiting faculty were available for the postexpedition analysis time frame to guide the manipulation of data collected from the field, data cleaning, and data analyses. Students presented preliminary posters to the Woodwell and Woods Hole research community and continued to analyze data in preparation for poster presentations at the AGU conference in December.

\section{Educational Theory and Assessment}

Self-efficacious experiences or involvements that communicate to individuals their ability to organize and execute courses of action necessary to be successful in a particular vocation are thought to increase a person's interest in said career (Bandura 1986; Lent et al. 2008; Wu 2018). Selfefficacy, a domain-specific construct, has been explored to understand whether and how it increases during various occurrences such as classroom and research experiences. Research on self-efficacy has established that the strongest relationships between self-efficacy and positive outcomes emerge when specific forms of self-efficacy are matched with specific outcomes (Choi 2005; Pajares and Miller 1995). In other words, it is important to examine selfefficacy for specific involvements with relevant outcomes for those involvements and not general self-efficacy. This study explored the specific construct of Arctic scientific self-efficacy, or self-efficacy associated with conducting Arctic and climate science research.

Numerous studies report positive relationships between scientific self-efficacy and positive outcomes such as persistence in STEM fields (Britner 2002; Britner and Pajares 2001, 2006; Lent et al. 2008) and aspirations for or participation in graduate research experiences and careers (Adedokun, Bessenbacher, et al. 2013; Adedokun, Zhang, et al. 2012; Livinti, Gunnesch-Luca, and Iliescu, 2021). Although self-efficacy-related analyses are widely found for traditional research experiences and in fields less interdisciplinary in nature, few studies have considered immersive research and field experiences in the geosciences (Dykas and Valentino 2016; Kortz, Cardace, and Savage 2020; Pfeifer et al. 2021; Streule and Craig 2016; Trott et al. 2020). This study aimed to explore the application of self-efficacy-building experiences in the immersive field excursion associated with the Polaris Project.

Studies exploring the processes and contextual and participant factors associated with the positive relationship between scientific self-efficacy and research participation suggest that research skills are mediated through self-efficacy beliefs (Adedokun, Bessenbacher, et al. 2013; Adedokun, Zhang, et al. 2012; Berkes 2007). Berkes argued that research efficacy beliefs were derived from mastery experiences, one of four types of involvement often associated with increases in self-efficacy. Three other involvements (vicarious experiences, verbal persuasions, and emotional/ physiological states) are thought to combine with mastery experiences to generate self-efficacy increases. However, mastery experiences are thought to have the greatest impact on self-efficacy (Bandura 1997). For this reason, this study focused on mastery experiences only. In the following section, mastery experiences are defined, followed by a brief overview of how mastery experiences interact and the justification for exploring them in this analysis of the Polaris Project.

Mastery experiences, most often based on previous encounters and execution of specific tasks, are defined when one successfully completes interesting, thought-provoking, and challenging vocation-related tasks that include well-planned activities that control for unproductive negative emotional and physiological arousals (Bandura 1997; Carter 2011). Research suggests that mastery experience tasks should be completed over an extended period with experts modeling the appropriate behaviors necessary to complete the task (Bandura 1997). Recipients of mastery experiences should receive instruction to guide individual performance and joint performance with experts to reinforce a sense of personal efficacy (Carter 2011).

With the goal of understanding the influence of self-efficacy on career aspirations in the face of limited understanding of the construct in relation to immersive, geoscience field research-based experiences, the 2016 Polaris PIs examined the presence of self-efficacy-building experiences in the Polaris program. Additionally, the study aimed to provide insight into any increases in awareness and understanding of Arctic career options potentially associated with self-efficacy-building experiences.

The interdisciplinary nature of Arctic science research, the Polaris Project's design and purpose, and the immersive and collaborative research expedition presented a culturally and scientifically complex assessment scenario. Assessment scenarios involving multiple participant groups and outcomes and limited explanatory mechanisms suggest the need for mixed-methods and participatory assessment approaches. The mixed-methods and participatory assessment approach was implemented in several steps. First, the assessment team included two researchers of color with expertise in behavioral and educational disciplines and experience identifying and assessing self-efficacy in undergraduate research settings to explain the benefits of research participation of underrepresented minorities in STEM. These researchers embodied lived experiences 
TABLE 2. Polaris Outcomes of Interest with Relevant Interview Questions

\begin{tabular}{|l|l|}
\hline Polaris outcomes of interest & \multicolumn{1}{|c|}{ Interview question } \\
\hline Career goals & $\begin{array}{l}\text { Please describe your 5-year and 10-year educational and/or career plans. } \\
\text { Probe: What are your thoughts on becoming an Arctic scientist? }\end{array}$ \\
\hline Mastery experience: interesting & What is the most interesting thing you did during your summer research experience? \\
\hline Mastery experience: challenging & Please describe your most challenging experience as a Polaris participant and how you dealt with it. \\
\hline Verbal or social persuasion & $\begin{array}{l}\text { What type of feedback have you gotten on your fieldwork, data analysis, or research project? What type } \\
\text { of feedback have you gotten from professors, peers, and family as a result of the Polaris Project? }\end{array}$ \\
\hline Vicarious experience & $\begin{array}{l}\text { Can you describe any Arctic researcher's work that you admire the most? Do you consider that person a } \\
\text { role model? Are there any Arctic researchers that you consider a role model? How do you think you are } \\
\text { similar to that person? }\end{array}$ \\
\hline Physiological state & \begin{tabular}{l} 
Tell me about your emotional state during various parts of the project. \\
\hline
\end{tabular}
\end{tabular}

by participating in and studying research experiences in STEM as diverse scientists who had ties to minorityserving institutions. Second, prior to the immersive field experience, the evaluation team participated in the Polaris Project Spring Orientation and online sessions with students to understand and observe implementation of the design principles. Third, program participants were regularly encouraged to develop their own research projects, hypotheses, and designs and to use mentors to support completion of their projects. Faculty also were encouraged to (1) support the detailed technical needs of their students' projects; (2) guide participants to consider the broader implications of Arctic science; and (3) explore linkages between their scientific major or area of focus and Arctic science. This approach resulted in a partnership among the researchers, the PIs, and the participants to inform and collect rich data about the meaning of the participant's experiences.

In year 1 of the program, the PIs did not receive training on how to build mastery experiences. However, the program was assessed for self-efficacy traits. The year 1 assessment reports included detailed information on the components of mastery and other self-efficacy experiences. The program was modified to intentionally incorporate those experiences in year 2 of the program.

This study was limited to the participants' involvement in one cohort-based extended research experience. Although students were offered multiyear interactions, the qualitative data analyzed and reported in this article assesses only experiences that occurred in the first year of participation. Analyses of the qualitative participant interview data represented the first component of the mixed-methods research process. Although a longitudinal survey is planned to collect quantitative data from all undergraduate and graduate participants, generalization of the findings will be limited given that each cohort includes only 12 undergraduate and graduate students. The potential for robust quantitative analysis from the Polaris Project is limited. These limitations further justify the focus on qualitative data to assess the Polaris program and to examine the processes of research experiences, the benefits of those processes, and their influence on students' career aspirations.

\section{Methodology}

To assess the presence of self-efficacy-building experiences and explore the potential influence of those experiences on participants' self-efficacy and career goals, a mixed-methods, participatory assessment approach was implemented in several steps. The evaluation study with relevant data collection instruments was approved by the Massachusetts General Hospital Institutional Review Board. The relevant data collection instruments were interview protocols for interviews utilized during orientation (baseline interviews), during the research expedition (midpoint interviews), and at the American Geophysical Union Fall Meeting where participants' research posters were presented (end of year 1 interviews). The baseline, midpoint, and endpoint interview protocols were developed by the assessment team. Self-efficacy prompts included in the interview protocols are listed in the interview questions in Table 2. The interview questions followed standard procedures for self-efficacy research by asking about respondents' confidence or experiences associated with completing a given task.

For each cohort, interviews were conducted as planned, at orientation (baseline), during the research expedition and data analysis camp (midpoint), and during the American Geophysical Union Fall Meeting (endpoint). Interviews were conducted for the 2017, 2018, and 2019 Polaris project cohorts. However, this article only reports results from the 2017 and 2018 cohorts.

\section{Data Analysis}

The data were evaluated qualitatively by members of the Polaris Project assessment team. Interviews were 
transcribed in Trint, an online transcription software. Nvivo, a qualitative software package, was used to analyze the data. Responses were coded first by question, grouped by response types, labeled, and then analyzed both quantitatively and qualitatively. For example, reasons for choosing a STEM major were coded as "reason for major." The responses were then grouped by comments that indicated "enjoyment" and "other." Keywords from the definitions and question prompts associated with the four types of involvement that result in increases in self-efficacy were used to code participants' responses. For mastery experiences, a participant's response was coded as a mastery experience if the respondent described the involvement as both interesting and challenging. Because previous studies classify completing a research poster as a mastery experience, respondents' descriptions of completion of a research poster were considered a mastery experience, and the analyses examined what respondents associated with those experiences.

Word clouds were chosen to illustrate self-efficacy-building experiences from the language respondents used to describe aspects of the Polaris Project and to characterize whether language suggested the presence of self-efficacybuilding experiences. Different word clouds of participant language from midpoint and endpoint interviews were developed to illustrate language and emerging themes for the field experience and data analysis camp and the poster presentation, respectively.

In the process of analyzing the language of Polaris participants, it was hypothesized that the design and structure of the Polaris Project allowed for participants to have self-efficacy-building experiences and to increase awareness and understanding of Arctic career options. Similarly, by exposure to various aspects of Arctic research, it is anticipated that Polaris participants will serve as climate change advocates with broad public audiences; however, this has yet to be explored in assessment of the program's outcomes.

Although this study did not explore the influence of group mentoring on participants' experiences, it was thought that the interdisciplinary demands of climate change research would negatively interact with the benefits of an individual mentor for Polaris participants. The question remains: As participants did not have an individual mentor in Polaris, did they feel lost or disconnected? Interview questions did not address this aspect of participants' experiences. However, the longitudinal survey and future Polaris projects should consider incorporating items to better assess this aspect of the Polaris Project. Two potential questions may include the following: (1) What are the impacts of having several mentors for mentees as they participate in the Polaris Project? (2) What is the implication of having multiple mentors for future career prospects in Arctic science research? Planned future analyses of the Polaris Project will examine data for all three cohorts as well as explore the benefits of the extended mentoring relationship. The Polaris program can be more attentive to mentoring by providing mentors training on the following: (1) building self-efficacy in trainees; (2) valuing culture in research experiences; and (3) promoting intersectionality in geoscience research, which will increase understanding and benefits from the extended mentoring relationships.

\section{Demographics and Academic Disciplines}

Table 3 illustrates that the Polaris Project included students of multiple racial and ethnic groups, academic levels, and climate science academic disciplines. Gender and racial/ethnic groups, including Native Americans, Hispanics, and African Americans, who have traditionally been underrepresented in Arctic science research represented over 50 percent of Polaris participants. Moreover, 75 percent of Polaris participants identified as female. Polaris participants spanned all levels of higher education from first-year undergraduates to graduate students. Both the 2017 and 2018 cohorts consisted mostly of upper-level undergraduate students, with fourth-year students representing one-third of the participants. Although third-year students composed 50 percent of the cohort in 2017, participants were spread across all academic levels in 2018. In terms of academic discipline, the 2017 cohort featured participants mostly from biology (33 percent) and hydrology (25 percent), whereas the 2018 cohort was made up mostly of environmental science (58 percent) and ecology (17 percent) majors. In each cohort of students, the majority reported aspirations to obtain an advanced degree in STEM during baseline interviews.

\section{Self-Efficacy Experiences and Outcomes}

The Polaris Project upholds the goal of providing inclusive experiences for both undergraduate and graduate students. Therefore, to maintain anonymity of the participants, the results are aggregated and do not distinguish between undergraduate and graduate students. Themes and language emerging from the student interview data suggest that components of the Polaris Project align with activities that provide mastery experiences. All Polaris participants expressed interest in their activities, challenges, stress, and personal success for the three major components of the Polaris Project: the field experience, the data analysis camp, and the poster presentation. All participants described the major components as interesting or fun but also reported stressful experiences during fieldwork. Likewise, although most participants reported that the data analysis period was stressful, some participants enjoyed it. To convey the language used by participants to describe the interesting and challenging aspects of the field experience and data analysis camp, midpoint interviews for 2017 and 2018 were combined into a word cloud shown in Figure 2. The most frequently used words appear the 
TABLE 3. Descriptive Statistics of Polaris Participants for 2017 and 2018

\begin{tabular}{|c|c|c|}
\hline Demographics & $2017(n=12)$ & $2018(n=12)$ \\
\hline \multicolumn{3}{|l|}{ Gender } \\
\hline Male & $25 \%$ & $25 \%$ \\
\hline Female & $75 \%$ & $75 \%$ \\
\hline \multicolumn{3}{|l|}{ Race/ethnicity } \\
\hline White & $42 \%$ & $34 \%$ \\
\hline Underrepresented minorities & $58 \%$ & $66 \%$ \\
\hline Native American & $25 \%$ & $25 \%$ \\
\hline Hispanic & $25 \%$ & $17 \%$ \\
\hline Asian & $8 \%$ & $8 \%$ \\
\hline African American & $0 \%$ & $8 \%$ \\
\hline Other/Multiracial & $0 \%$ & $8 \%$ \\
\hline \multicolumn{3}{|l|}{ Classification } \\
\hline First-year undergraduate & $0 \%$ & $8 \%$ \\
\hline Second-year undergraduate & $0 \%$ & $17 \%$ \\
\hline Third-year undergraduate & $50 \%$ & $18 \%$ \\
\hline Fourth-year undergraduate & $33 \%$ & $33 \%$ \\
\hline Master's student & $0 \%$ & $16 \%$ \\
\hline PhD student & $17 \%$ & $8 \%$ \\
\hline \multicolumn{3}{|l|}{ Major } \\
\hline Biology & $33 \%$ & $9 \%$ \\
\hline Ecology & $0 \%$ & $17 \%$ \\
\hline Education & $0 \%$ & $8 \%$ \\
\hline Environmental science ${ }^{a}$ & $17 \%$ & $58 \%$ \\
\hline Forestry & $8 \%$ & $0 \%$ \\
\hline Geology & $8 \%$ & $0 \%$ \\
\hline Hydrology & $25 \%$ & $8 \%$ \\
\hline Natural science & $9 \%$ & $0 \%$ \\
\hline \multicolumn{3}{|l|}{ Academic goals } \\
\hline $\mathrm{PhD}$ & $75 \%$ & $50 \%$ \\
\hline Unknown & $8 \%$ & $25 \%$ \\
\hline Master's & $9 \%$ & $17 \%$ \\
\hline Bachelor & $0 \%$ & $8 \%$ \\
\hline MD & $8 \%$ & $0 \%$ \\
\hline
\end{tabular}

Note: ancludes global environmental change and Earth and planetary sciences

largest in the cloud and included words such as amazed, great, excited, and pretty. Other frequently used words appearing in the cloud included words such as people, field, experience, project, and done. Words that related to
FIGURE 2. Word Cloud of Participant Field and Data Camp Language for 2017 and 2018: Midpoint Interviews

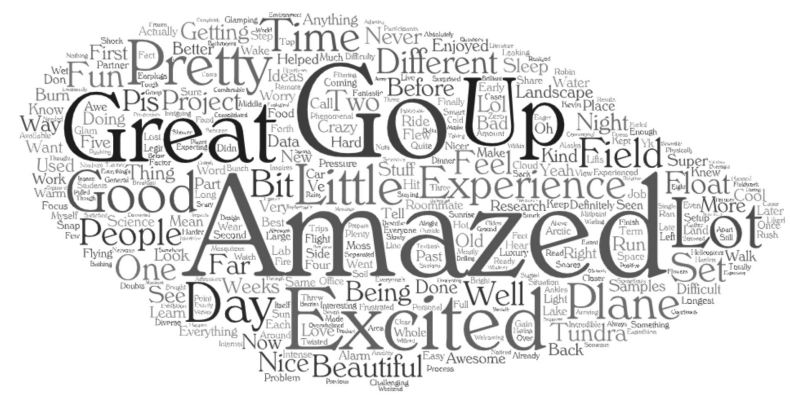

FIGURE 3. Word Cloud of Participant Field and Data Camp Language for 2017 and 2018: Endpoint Interviews

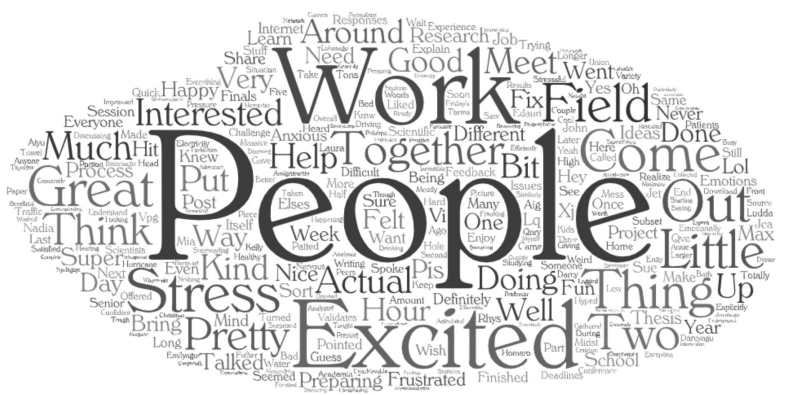

challenging experiences included worry, crazy, and hard and appear in smaller type because they were mentioned less frequently.

Figure 3 illustrates the language participants used to describe the poster presentation component of their Polaris experiences in endpoint interviews in 2017 and 2018. Although some participants reported stress during the preparation phase, all participants described enjoying presenting the posters. As described, mastery experiences involve the execution of specific tasks that may be described as interesting, thought provoking, and challenging. Words such as stress, interested, and think were prominently used by participants to describe the poster presentation process, suggesting that this activity aligned with mastery experiences.

\section{Arctic Career Awareness}

As mentioned earlier, a broader objective of the Polaris Project is to motivate and sustain participation in climate or Arctic science research careers. To this end, data were collected about participants' experiences with the field expedition, their experiences analyzing and presenting data, and their awareness of and aspiration for Arctic careers. Participants were classified as having Arctic/climate science career goals if the words Arctic, climate, or 
FIGURE 4. Word Cloud of Participants' Career Goal Responses for 2017 and 2018: Baseline

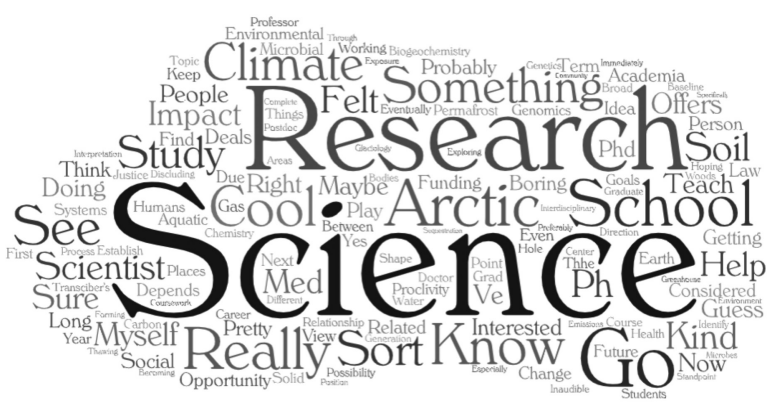

FIGURE 5. Word Cloud of Participants' Career Goal Responses for 2017 and 2018: Endpoint

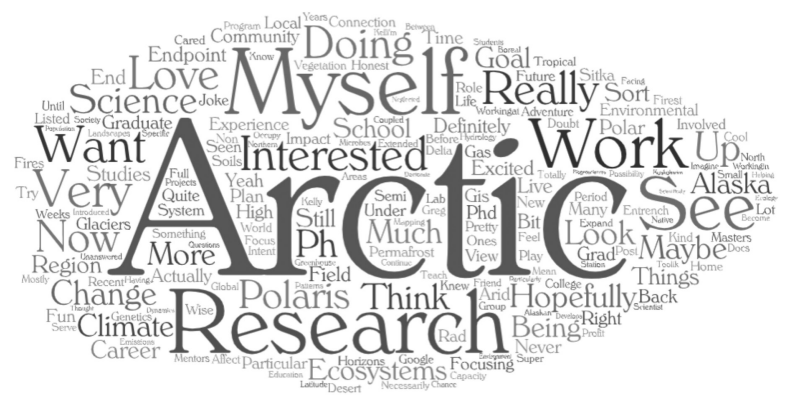

Alaska emerged when they were asked about their career goals. Participants were classified as having non-Arctic/ climate career goals if they articulated a career goal but did not mention the words Arctic, climate, Alaska, or any words pertaining to Arctic science. Lastly, participants were classified as undecided if their responses excluded words related to Arctic science and included the words, $I$ don't know, anywhere, or not sure.

The percent of participants using language about Arctic careers more than doubled in 2017 , from 25 percent at baseline to 73 percent at endpoint, and in 2018, from 33 percent at baseline to 70 percent at endpoint. The word cloud in Figure 4 shows an overall increase in the use of language about Arctic science careers across all students. Comparing Figure 4 (baseline) to Figure 5 (endpoint), it is seen that the word Arctic is more prominent, indicating more frequent mention at endpoint than baseline, when Arctic is present but smaller. This suggests an increased awareness and understanding of Arctic career options.

\section{Challenges and Lessons Learned}

The Polaris Project overcame challenges associated with immersive and remote field research-based experiences such as addressing logistical arrangements, personal health needs, and interpersonal conflicts. The principal investigators and project staff attempted to address these challenges by building relationships and collaborations on personal and professional levels before the remote experience as well as having appropriately trained medical staff and equipment on site.

Regarding research experiences, challenges were identified with conducting fieldwork, traveling, and delivering a research product in the summer. From a self-efficacy standpoint, mastering these challenging experiences may be considered opportunities for participant growth. However, strategies to balance stress and growth are necessary when designing intense and immersive experiences for future programs. Presenting experiences theorized to have an impact on interest in climate change careers in participants in an Arctic science undergraduate research program resulted in an effective interdisciplinary model for teaching about climate change impacts that allowed for self-efficacy-building experiences, increased awareness among participants about climate change research careers, and participation in climate change advocacy.

The impact on climate and Arctic science facilitated by the Polaris Project is successful because it allows students to apply their scientific interests and cultural experiences toward solving a research problem. The model allows for interdisciplinary experiences that provide broad knowledge of climate change research and its impacts. Because of the diverse representation of participants, application and knowledge transfer to others in communities currently underrepresented in climate change research, work, and advocacy are anticipated. Disseminating knowledge to underrepresented communities will allow for far-reaching approaches to combat the harmful impacts of climate change. Programs interested in providing self-efficacybuilding experiences and increasing awareness of climate change research and careers should consider deeply immersing students in intellectually and physically challenging environments to address scientifically and socially urgent topics. This immersion is critical to stimulation and widespread implementation of the diverse, innovative, and impactful climate change solutions necessary to change the current course of the planet.

\section{Acknowledgments}

The research discussed in this article was conducted as part of the Polaris Project, a National Science Foundationfunded program (NSF award 1624927). Although author Frances Carter-Johnson is an employee of the federal government, any views expressed are those of the authors and not necessarily those of the National Science Foundation.

\section{References}

Adedokun, Omolola A., Ann B. Bessenbacher, Loran C. Parker, Lisa L. Kirkham, and Wilella D. Burgess. 2013. "Research Skills and STEM Undergraduate Research Students' Aspirations for 
Research Careers: Mediating Effects of Research Self-Efficacy." Journal of Research in Science Teaching 50: 940-951. doi: $10.1002 /$ tea.21102

Adedokun, Omolola A., Dake Zhang, Loran Carleton Parker, Ann Bessenbacher, Amy Childress, and Wilella Daniels Burgess. 2012. "Understanding How Undergraduate Research Experiences Influence Student Aspirations for Research Careers and Graduate Education." Journal of College Science Teaching 42(1): 82-90.

Adetunji, Oludurotimi O., Jean-Claude M. Ba, Woldai Ghebreab, Jonathan F. Joseph, Laurence P. Mayer, and Roger Levine. 2012. "Geosciences Awareness Program: A Program for Broadening Participation of Students in Geosciences." Journal of Geoscience Education 60: 234-240. doi: 10.5408/10-208.1

Atkins, Kaitlyn, Bryan M. Dougan, Michelle S. Dromgold-Sermen, Hannah Potter, Viji Sathy, and A. T. Panter. 2020. "'Looking at Myself in the Future': How Mentoring Shapes Scientific Identity for STEM Students from Underrepresented Groups." International Journal of STEM Education 7: ar. 42. doi: 10.1186/ s40594-020-00242-3

Bandura, Albert. 1986. Social Foundations of Thought and Action. Englewood Cliffs, NJ: Prentice Hall.

Bandura, Albert. 1997. Self-Efficacy: The Exercise of Control. New York: Freeman.

Berkes, Elizabeth. 2007. "Practicing Biology: Undergraduate Laboratory Research, Persistence in Science, and the Impact of Self-Efficacy Beliefs." PhD dissertation, Washington University in St. Louis.

Bradley, Evan D., Michelle Bata, Heather M. Fitz Gibbon, Caroline J. Ketcham, Brittany A. Nicholson, Meagen Pollock, and Erin Dolan. 2017. "Structure of Mentoring in Undergraduate Research: Multi-Mentor Models." Scholarship and Practice of Undergraduate Research 1(2): 35-42. doi: 10.18833/spur/1/2/12

Britner, Shari Lynn. 2002. "Science Self-Efficacy of African American Middle School Students: Relationship to Motivation Self-Beliefs, Achievement, Gender, and Gender Orientation." $\mathrm{PhD}$ dissertation, Emory University.

Britner, Shari L., and Frank Pajares. 2001. "Self-Efficacy Beliefs, Motivation, Race, and Gender in Middle School Science." Journal of Women and Minorities in Science and Engineering 7(4): 271-285. doi: 10.1615/jwomenminorscieneng.v7.i4.10

Britner, Shari L., and Frank Pajares. 2006. "Sources of Science Self-Efficacy Beliefs of Middle School Students." Journal of Research in Science Teaching 43: 485-499. doi: 10.1002/tea.20131

Callahan, Caitlin N., Nicole D. LaDue, Lorenzo D. Baber, Julie Sexton, Katrien J. van der Hoeven Kraft, and Eboni M. Zamani-Gallaher. 2017. "Theoretical Perspectives on Increasing Recruitment and Retention of Underrepresented Students in the Geosciences." Journal of Geoscience Education 65: 563-576. doi: $10.5408 / 16-238.1$

Callahan, Caitlin N., Julie C. Libarkin, Carmen M. McCallum, and Christopher L. Atchison. 2015. "Using the Lens of Social Capital to Understand Diversity in the Earth System Sciences Workforce." Journal of Geoscience Education 63: 98-104. doi: 10.5408/15-083.1
Carter, Frances D. 2011. "An Analysis of Scientific Self-Efficacy as a Benefit of Summer Research Participation for Underrepresented Minorities in Science, Technology, Engineering, and Mathematics (STEM) Fields." PhD dissertation, University of Maryland, Baltimore County.

Choi, Namok. 2005. "Self-Efficacy and Self-Concept as Predictors of College Students' Academic Performance." Psychology in the Schools 42: 197-205. doi: 10.1002/pits.20048

Dykas, Matthew J., and David W. Valentino. 2016. "Predicting Performance in an Advanced Undergraduate Geological Field Camp Experience." Journal of Geoscience Education 64: 314-322. doi: 10.5408/15-128.1

Garrett, Arnell Trudy, and Frances Carter-Johnson. 2019. "Learning Experiences That Led to Climate Science Career Decisions: The Polaris Project Arctic Science Social Cognitive Career Framework." AGU Fall Meeting Abstracts 2019: ED54B-03.

Huntoon, Jacqueline E., and Melissa J. Lane. 2007. "Diversity in the Geosciences and Successful Strategies for Increasing Diversity." Journal of Geoscience Education 55: 447-457. doi: 10.5408/1089-9995-55.6.447

Joshi, Megha, Melissa L. Aikens, and Erin L. Dolan. 2019. "Direct Ties to a Faculty Mentor Related to Positive Outcomes for Undergraduate Researchers." BioScience 69: 389-397. doi: 10.1093/biosci/biz039

Kortz, Karen M., Dawn Cardace, and Brian Savage. 2020. "Affective Factors during Field Research That Influence Intention to Persist in the Geosciences." Journal of Geoscience Education 68: 133-151. doi: 10.1080/10899995.2019.1652463

Lent, Robert W., Hung-Bin Sheu, Daniel Singley, Janet A. Schmidt, Linda C. Schmidt, and Clay S. Gloster. 2008. "Longitudinal Relations of Self-Efficacy to Outcome Expectations, Interests, and Major Choice Goals in Engineering Students." Journal of Vocational Behavior 73: 328-335. doi: 10.1016/j. jvb.2008.07.005

Livinţi, Raluca, George Gunnesch-Luca, and Dragoș Iliescu. 2021. "Research Self-Efficacy: A Meta-Analysis." Educational Psychologist 56: 215-242. doi: 10.1080/00461520.2021.1886103

Mattheis, Allison, Megan Murphy, and Erika Marin-Spiotta. 2019. "Examining Intersectionality and Inclusivity in Geosciences Education Research: A Synthesis of the Literature, 20082018." Journal of Geoscience Education 67: 505-517. doi: $10.1080 / 10899995.2019 .1656522$

McDaris, John R., Cathryn A. Manduca, Ellen R. Iverson, and Cailin Huyck Orr. 2017. "Looking in the Right Places: Minority-Serving Institutions as Sources of Diverse Earth Science Learners." Journal of Geoscience Education 65: 407-415. doi: 10.5408/16-224.1

National Academies of Sciences, Engineering, and Medicine. 2019. The Science of Effective Mentorship in STEMM. Washington, DC: National Academies Press.

Nicholson, Brittany A., Meagen Pollock, Caroline J. Ketcham, Heather M. Fitz Gibbon, Evan D. Bradley, and Michelle Bata. 2017. "Beyond the Mentor-Mentee Model: A Case for Multi-Mentoring in Undergraduate Research." Perspectives on Undergraduate Research and Mentoring 6: 1-14. Accessed August 15, 2021. 
https://www.elon.edu/u/academics/undergraduate-research/purm/ wp-content/uploads/sites/923/2019/06/Nicholson_et_al_6.1.pdf

Pajares, Frank, and M. David Miller. 1995. "Mathematics SelfEfficacy and Mathematics Performances: The Need for Specificity of Assessment." Journal of Counseling Psychology 42: 190-198. doi: 10.1037/0022-0167.42.2.190

Pastick, Neal J., M. Torre Jorgenson, Bruce K. Wylie, Shawn J. Nield, Kristofer D. Johnson, and Andrew O. Finley. 2015. "Distribution of Near-Surface Permafrost in Alaska: Estimates of Present and Future Conditions." Remote Sensing of Environment 168: 301-315.

Peppoloni, Silvia, and Giuseppe Di Capua. 2016. "Geoethics: Ethical, Social, and Cultural Values in Geosciences Research, Practice, and Education." In Geoscience for the Public Good and Global Development: Toward a Sustainable Future, ed. Gregory R. Wessel and Jeffrey K. Greenberg, 17-21. Geological Society of America Special Papers, Vol. 520. Boulder, CO: Geological Society of America. doi: 10.1130/2016.2520(03)

Pfeifer, Lily S., Michael J. Soreghan, Kelly K. Feille, Gerilyn S. Soreghan, Gary S. Weissmann, Robert A. Ibarra, and Wesley A. Stroud. 2020. "Activation of the Multicontext Model in a Field-Based Program for Traditionally Underserved Students." Journal of Geoscience Education 69: 85-95. doi: $10.1080 / 10899995.2020 .1838850$

Saito, Kazuyuki, Tingjun Zhang, Daqing Yang, Sergei Marchenko, Roger G. Barry, Vladimir Romanovsky, and Larry Hinzman. 2013. "Influence of the Physical Terrestrial Arctic in the EcoClimate System.” Ecological Applications 23: 1778-1797.

Sherman-Morris, Kathleen, and Karen S. McNeal. 2016. "Understanding Perceptions of the Geosciences among Minority and Nonminority Undergraduate Students." Journal of Geoscience Education 64: 147-156. doi: 10.5408/15-112.1

Streule, Mike, and Lorraine Craig. 2016. "Social Learning Theories: An Important Design Consideration for Geoscience Fieldwork." Journal of Geoscience Education 64: 101-107. doi: $10.5408 / 15-119.1$

Trott, Carlie D., Laura B. Sample McMeeking, Cheryl L. Bowker, and Kathryn J. Boyd. 2020. "Exploring the Long-Term Academic and Career Impacts of Undergraduate Research in Geoscience: A Case Study." Journal of Geoscience Education 68: 65-79. doi: 10.1080/10899995.2019.1591146

Wolfe, Benjamin A., and Eric M. Riggs. 2017. "Macrosystem Analysis of Programs and Strategies to Increase Underrepresented
Populations in the Geosciences." Journal of Geoscience Education 65: 577-593. doi: 10.5408/17-256.1

Wu, Ben H. 2018. "The Role of Career Optimism and Perceived Barriers in College Students' Academic Persistence: A Social Cognitive Career Theory Approach.” PhD dissertation, University of Southern Mississippi.

\section{Frances D. Carter-Johnson}

National Science Foundation, frajohns@nsf.gov

Arnell Garrett-an independent policy researcher, evaluator, and founder of Delmarva Analysis, LLC-earned a $B A$ from the University of North Carolina at Chapel Hill and an MPP from the University of Maryland, Baltimore County, and has conducted analyses for leading nonprofit and professional society organizations such as AARP and the American Institute of Physics.

Frances D. Carter-Johnson is an education data scientist in the Directorate for Education and Human Resources, Division of Human Resource Development, at the National Science Foundation, where she is responsible for assessing outcomes of broadening participation programs and contributing to the body of STEM education policy research and knowledge.

Susan M. Natali is an associate scientist at the Woodwell Climate Research Center, where her research focuses on the impact of climate change on terrestrial ecosystems, primarily on arctic permafrost.

The late John D. Schade served as a program officer in the Division of Environmental Biology at the National Science Foundation (NSF), where he managed the Ecosystem Science and Long-Term Ecological Research programs. Before his time at NSF, he spent a sabbatical year at the Woodwell Climate Research Center and 10 years on the faculty at St. Olaf College.

Robert Max Holmes is a senior scientist and deputy director at the Woodwell Climate Research Center. Conducting field research throughout Siberia, Alaska, and the Canadian Arctic, he has contributed toward understanding how a changing climate will impact Arctic rivers, watersheds, and permafrost. 